\title{
Specific features of eddy turbulence in the turbopause region
}

\author{
M. N. Vlasov and M. C. Kelley \\ School of Electrical and Computer Engineering, Cornell University, Ithaca, NY, USA \\ Correspondence to: M. N. Vlasov (mv75@cornell.edu)
}

Received: 20 March 2013 - Revised: 12 December 2013 - Accepted: 11 February 2014 - Published: 15 April 2014

\begin{abstract}
The turbopause region is characterized by transition from the mean molecular mass (constant with altitude) to the mean mass (dependent on altitude). The former is provided by eddy turbulence, and the latter is induced by molecular diffusion. Competition between these processes provides the transition from the homosphere to the heterosphere. The turbopause altitude can be defined by equalizing the eddy and molecular diffusion coefficients and can be located in the upper mesosphere or the lower thermosphere. The height distributions of chemical inert gases very clearly demonstrate the transition from turbulent mixing to the diffusive separation of these gases. Using the height distributions of the chemical inert constituents $\mathrm{He}, \mathrm{Ar}$, and $\mathrm{N}_{2}$ given by the MSIS-E-90 model and the continuity equations, the height distribution of the eddy diffusion coefficient in the turbopause region can be inferred. The eddy diffusion coefficient always strongly reduces in the turbopause region. According to our results, eddy turbulence above its peak always cools the atmosphere. However, the cooling rates calculated with the eddy heat transport coefficient equaled to the eddy diffusion coefficient were found to be much larger than the cooling rates corresponding to the neutral temperatures given by the MSIS-E90 model. The same results were obtained for the eddy diffusion coefficients inferred from different experimental data. The main cause of this large cooling is the very steep negative gradient of the eddy heat transport coefficient, which is equal to the eddy diffusion coefficient if uniform turbulence takes place in the turbopause region. Analysis of wind shear shows that localized turbulence can develop in the turbopause region. In this case, eddy heat transport is not so effective and the strong discrepancy between cooling induced by eddy turbulence and cooling corresponding to the temperature given by the MSIS-E-90 model can be removed.
\end{abstract}

Keywords. Atmospheric composition and structure (middle atmosphere - composition and chemistry) - meteorology and atmospheric dynamics (middle atmosphere dynamics; turbulence)

\section{Introduction}

The turbopause region is characterized by transition from the mean molecular mass, constant with altitude, to the mean mass dependent on altitude. The former is provided by eddy turbulence, and the latter is induced by molecular diffusion. A competition between these processes provides the transition from the homosphere to the heterosphere. The turbopause altitude can be defined by equalizing the eddy and molecular diffusion coefficients and can be located in the upper mesosphere or the lower thermosphere. An example of the height distributions of eddy and molecular diffusion coefficients is shown in Fig. 1.

The densities of the main and minor constituents and total atmospheric density at all altitudes in the thermosphere strongly depend on the processes in the turbopause region. However, there is significant uncertainty in our understanding of turbulence in the turbopause. This uncertainty is partly induced by studying turbulence separately from estimates of the effects of turbulence on the main atmospheric parameters: the temperature, densities of neutral constituents, and charged particles. Vlasov and Kelley $(2010,2012)$ showed the importance of studying turbulence, together with its effects on temperature and atomic oxygen. First they found that eddy turbulence can provide an effective mechanism to explain the cold summer and warm winter mesopause observed at high latitudes, together with the seasonal variations of atomic oxygen. 


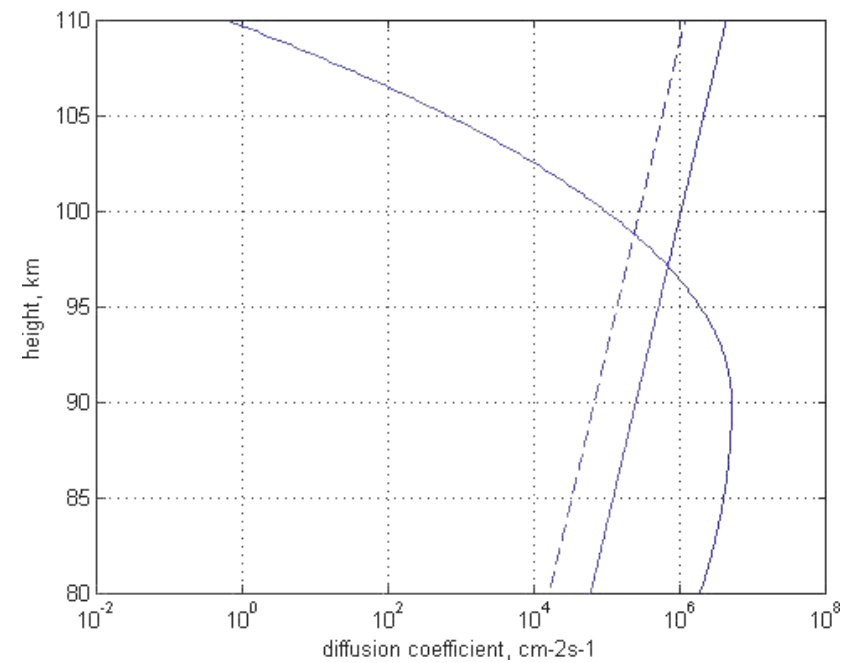

Fig. 1. Height distributions of molecular diffusion coefficients for $\mathrm{Ar}$ and $\mathrm{He}$ (dashed and solid lines, respectively) and the eddy diffusion coefficient (solid curve).

Although there is progress in estimating the eddy diffusion coefficient, significant uncertainty remains in determining the eddy diffusion coefficient. Thus, the experimental data and the theoretical estimates obtained during the period of 1970-1980 included maximum values of the eddy diffusion coefficient that were much larger than $1 \times 10^{7} \mathrm{~cm}^{2} \mathrm{~s}^{-1}$ (for example, Justus, 1973; Weinstock, 1984) with a very high peak altitude of $130 \mathrm{~km}$. The minima values were less by two orders of magnitude (Hocking, 1986). Also, very contradictory data on seasonal eddy diffusion variations existed (Blum and Schuchardt, 1978). Later, Vlasov and Korobeynikova (1991) showed that eddy diffusion with a coefficient larger than $5 \times 10^{6} \mathrm{~cm}^{2} \mathrm{~s}^{-1}$ induces a significant temperature height distribution deviation from empirical model distributions in the lower thermosphere. It was recognized that maximum values of the eddy diffusion coefficient occur during summer and at high latitudes. This significant advance was achieved due to the measurements of Fukao et al. (1994) and Lübken (1997), and we have used Lübken's measurements in this paper. More recent data on the eddy diffusion coefficient are discussed below.

In contrast to the statistical concept of the turbopause localized within the altitude range of $90-110 \mathrm{~km}$, we consider the turbopause as the region where, at any given time, the combination of turbulence and molecular diffusion can be complicated. For example, this is demonstrated by the appearance of long-lived meteor trails, as presented by Kelley at al. (2003). Also, the Turbulent Oxygen Mixing Experiment (TOMEX) (Bishop et al., 2004) showed that "unstable regions are well mixed, but the intermediate regions, in some cases, have very small energy dissipation rates". According to these results, eddy diffusion may be important at altitudes above the turbopause, located at $103 \mathrm{~km}$.
We must emphasize that the energy deposition rates and the eddy diffusion coefficient, $K_{\text {ed }}$, corresponding to the rates estimated in TOMEX (see Tables 1 and 2 in Bishop et al., 2004), are larger than the parameters estimated by Lübken (1997) by a factor of $4-8$, and the $K_{\text {ed }}$ maximum value is larger than $1 \times 10^{7} \mathrm{~cm}^{2} \mathrm{~s}^{-1}$. However, the energy deposition rate, estimated from the density fluctuation measurements carried out during the rocket experiment (Szewczyk et al., 2013), is larger by a factor of 20 than the value estimated by Lübken (1997). We will discuss these results in Sect. 4.

All of the experimental methods have limitations and require some theoretical assumptions. The main assumption is linear dependence of the eddy diffusion coefficient $K_{\text {ed }}$ on the energy dissipation rate. Problems with applying this fairly restrictive assumption were noted many times (for example, Lübken, 1997; Fritts and Luo, 1995; Hocking, 1999). The energy dissipation rate, $\varepsilon$, is a key parameter in determining the eddy diffusion coefficient, $K_{\text {ed }}$, from experimental data. Usually, the spectrum of density fluctuations calculated from experimental data is approximated using the theoretical model of Heisenberg (1948) and the inner scale, $l_{0}$, is determined. This parameter is related to the Kolmogorov microscale, $\eta$, through the relation $l_{0}=9.9 \eta$ (Lübken et al., 1993). The Kolmogorov microscale is a rough estimate of the size of the smallest eddies, which can provide the turbulent energy dissipation by viscosity $\nu$. Then the $\varepsilon$ value can be calculated using the formula $\varepsilon=v^{3} \eta^{-4}$. According to this formula, the $\varepsilon$ value strongly depends on the $\eta$ value, which is estimated by a rough approximation. For example, let us estimate the impact of $\eta$ values on the energy dissipation rate using the $l_{0}$ values inferred from the experimental data by Kelley et al. (2003). These values are changed from $156 \mathrm{~m}$ to $222 \mathrm{~m}$, and the $\varepsilon$ value can change from $0.14 \mathrm{~W} \mathrm{~kg}^{-1}$ to $0.58 \mathrm{~W} \mathrm{~kg}^{-1}$. The formula $K_{\mathrm{ed}}=b \varepsilon / \omega_{\mathrm{B}}^{2}(*)$ is used, and here $b$ is usually assumed to be constant and $\omega_{\mathrm{B}}$ is the buoyancy frequency. Kelley et al. (2003) estimated the $K_{\text {ed }}$-averaged value to be $500 \mathrm{~m}^{2} \mathrm{~s}^{-1}$. Taking into account the $\varepsilon$ variation estimated above, the $K_{\text {ed }}$ values can vary from $250 \mathrm{~m}^{2} \mathrm{~s}^{-1}$ to $1000 \mathrm{~m}^{2} \mathrm{~s}^{-1}$, which, except for the very highest values, correspond to measured $K_{\text {ed }}$ values in the mesosphere and lower thermosphere (Fukao et al., 1994).

The same problem exists in estimating the energy dissipation rate inferred from chemical tracer observations. In this case, formula $\varepsilon=r_{t}^{2} t^{-3} /(2.4 \alpha)^{1.5}$ (Rees et al., 1972, and references therein) is usually used. Here, $r_{t}$ is the trail radius as a function of time, $t$, and $\alpha$ is a Kolmogorov constant. The values of this constant vary between 0.5 and 1.5 because the absolute value is unknown (Weinstock, 1978; Rees et al., 1972). In this case, the $\varepsilon$ value can change by a factor of 5 due to the uncertainty of a Kolmogorov constant. Note that Bishop et al. (2004) had to use the $\alpha$ maximum value because the energy dissipation rates inferred from the chemical tracer dynamics were unusually high. We discuss the results of this experiment in Sect. 4. 
The other uncertainty results from determining the $b$ value in formula $(*)$. Usually, $b=0.81$ is used (Weinstock, 1978). However, formula $K_{\mathrm{ed}} \omega_{\mathrm{B}}^{2}(P-R i) / R i=\varepsilon$ where $P$ and $R i$ are the Prandtl and Richardson numbers, respectively, should be used according to Gordiets et al. (1982). The Prandtl number is equal to 1 for uniform turbulence and $R i=0.44$ for $b=$ 0.81 . The Kelvin-Helmholtz instability requires $R i \leq 0.25$, corresponding to $b=0.3$.

Using the electron density fluctuation spectra obtained by rocket-borne measurements of electron density at low latitudes, Das et al. (2009) showed that turbulence is not present continuously in the mesosphere but exists in layers of 100 $200 \mathrm{~m}$ interspersed with regions of stability.

Gravity waves, winds, and turbulence are the main dynamic processes in the upper mesosphere and the lower thermosphere (MLT). The source of these processes is uncertain, as is their role in the MLT energy budget. For example, Lübken et al. (1993) conclude that the impact of turbulence on the energy budget of the mesosphere and lower thermosphere is small. However, Lübken (1997) reconsidered their results and reported the importance of turbulence.

Gravity waves were first suggested by Hines (1960) to explain observed features in MLT. Then, the important role of gravity waves in the circulation, thermal balance, and constituent structures was recognized. Reviews of the work on gravity waves are given by Fritts (1984) and Fritts and Alexander (2003).

Gravity waves can transfer their energy and momentum into the MLT due to their dissipation via nonlinear interaction and wave-breaking processes (Weinstock, 1976). The turbulence can be generated in MLT (Lindzen, 1967; Hodges Jr., 1969). Heating can result from this dissipation (Becker, 2004; Medvedev and Klassen, 2003). Also, a downward heat flux can be induced during this dissipation, resulting in cooling. Medvedev and Klassen $(1995,2003)$ considered gravity wave dissipation due to nonlinear interaction across the wave spectrum and showed that there is cooling for saturated gravity waves in the upper portion of the MLT. However, there is a problem with estimating these effects, as described next.

It is assumed that the mean atmosphere is expected to be stable, both statically and dynamically, even in the presence of tides (Hodges Jr., 1967; Gardner et al., 2002). However, in the mesopause region where gravity waves can achieve high amplitudes, the combined effect of the background temperature profile, tides, and gravity waves can induce significantly large vertical shears in the horizontal wind and temperature profiles so that the atmosphere becomes unstable and the waves began to dissipate. Note that all of the experimental data on the height distribution of chemical inert gases show mixing of these gases at all altitudes below the mesopause.

It is assumed that eddy turbulence is due to convective or dynamic instability. The former develops when the negative temperature gradient is higher than the adiabatic lapse, and the latter occurs when the dynamic Richardson number, $R i$, is less than 0.25 . The Richardson number is defined by the ratio

$R i=\frac{\omega_{\mathrm{B}}^{2}}{S^{2}}$,

where $\omega_{\mathrm{B}}$ is the buoyancy frequency given by the formula

$\omega_{\mathrm{B}}^{2}=\frac{g}{T}\left(\frac{\partial T}{\partial z}+\frac{g}{C_{p}}\right)$,

where $T$ is the temperature, $g$ is the gravity acceleration, $C_{p}$ is the heat capacity of air at constant pressure, and

$S=\left[\left(\frac{\partial u}{\partial z}\right)^{2}+\left(\frac{\partial v}{\partial z}\right)^{2}\right]^{1 / 2}$

is the total vertical shear of the horizontal wind with zonal, $u$, and meridional, $v$, components.

In general, turbulence can be generated due to gravity waves, produced in the lower atmosphere, traveling upward and breaking at mesospheric heights and/or due to KelvinHelmholtz instability, which occurs in situ in the presence of strong wind shears. However, very intense shears would be required to meet the criterion $R i<0.25$ in the turbopause region. Also, the strong wind source is not clear.

The time of eddy diffusion can be estimated by the formula $\tau=H^{2} / K_{\text {ed }}=3.6 \times 10^{11} / 5 \times 10^{6}=7.2 \times 10^{4} \mathrm{~s} \approx 1$ day, meaning that significant fluctuations can exist within this time. Thus, fluctuations with a gradient scale of less than $2 \mathrm{~km}$ can take place during $2.2 \mathrm{~h}$. However, rocket measurements of the ratio $[\mathrm{Ar}] /\left[\mathrm{N}_{2}\right]$ show the continuity transition from the ratio corresponding to mixing (independent of altitude) to the altitude-dependent ratio corresponding to the diffusive separation of gases with different masses. Note that different experimental data show the mixing of atmospheric constituents below $80 \mathrm{~km}$ where conditions for the Kelvin-Helmholtz instability are not met because of low wind shear and high Brunt-Väisälä frequencies.

In this paper, using the height distributions of the chemical inert constituents $\mathrm{He}, \mathrm{Ar}$, and $\mathrm{N}_{2}$ given by the MSIS-E-90 model (Hedin, 1991) and the continuity equations, we infer the height distribution of the eddy diffusion coefficient in regions of transition from the homosphere to the heterosphere corresponding to the turbopause region. Then we show that heating/cooling by gravity waves can be described by eddy turbulence heat transport. We show that formulas for the eddy heating/cooling rate coincide with formulas for these rates obtained and used in estimating the thermal effect of gravity waves (Akmaev, 2007; Becker and McLandress, 2009, hereafter referred to as A07 and BM09). Using the eddy turbulence heating/cooling equation and assuming that the eddy heat transport coefficient is equal to the eddy diffusion coefficient, we calculate the cooling in the turbopause region. Note that eddy turbulence cools the atmosphere above the eddy diffusion coefficient peak, i.e., the turbopause region (see Fig. 1). Our results show that this cooling is much higher 


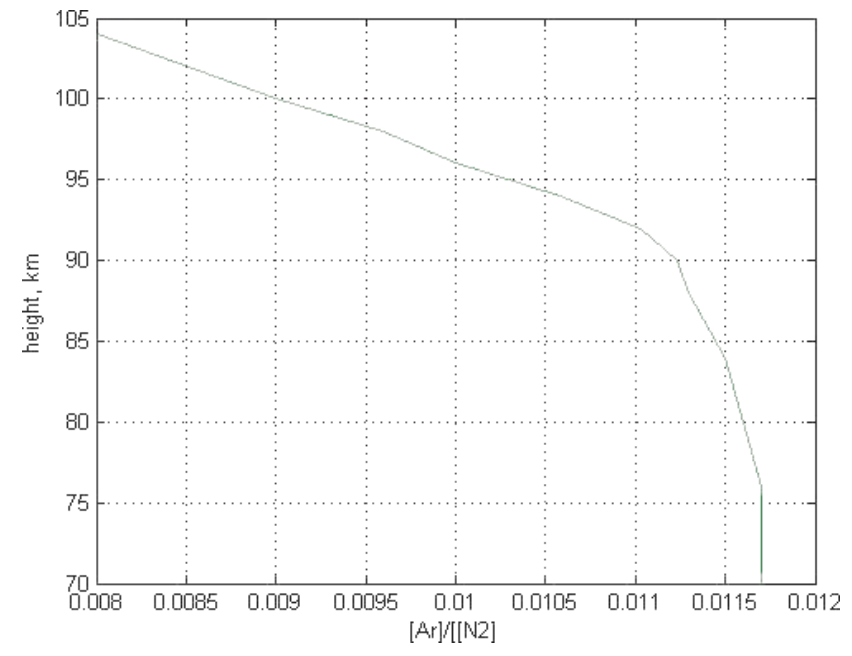

Fig. 2. Height profile of the $[\mathrm{Ar}] /\left[\mathrm{N}_{2}\right]$ ratio in summer solstice at a latitude of $40^{\circ} \mathrm{N}$ at 14:00 LT, given by the MSIS-E-90 model.

than normal cooling corresponding to temperatures given by the MSIS model at altitudes around the turbopause. This phenomenon results from strong cooling by eddy turbulence corresponding to a strong decrease in the eddy diffusion coefficient in the turbopause region (see Fig. 1). This high cooling means that the eddy heat transport coefficient should be smaller than the eddy diffusion coefficient.

The goal of this paper is to study the specific features of eddy turbulence in transition from the homosphere to the heterosphere under different geophysical conditions. Also, we analyze the conditions for developing eddy turbulence in the small temperature gradient region and below the main wind shear layer because, according to our results, the eddy turbulence peak occurs below the wind shear maximum and in regions with a positive or small negative temperature gradient.

In Sect. 2 we infer the height profile of the eddy diffusion coefficients using continuity equations and the height profiles of the chemical inert gases given by the MSIS-E-90 model. In Sect. 3 we estimate the cooling/heating profiles corresponding to the eddy heat transport coefficients equaled to the eddy diffusion coefficients estimated in Sect. 2. In Sect. 4, using the results obtained in previous sections and the eddy diffusion coefficients inferred from experimental data (Lübken, 1997; Bishop et al., 2004), we determine the specific features of eddy turbulence in the turbopause region and consider an instability mechanism in this region.

\section{Estimate of the eddy diffusion coefficient from height distributions of chemical inert gases given by the MSIS-E-90 model}

The $[\mathrm{Ar}] /\left[\mathrm{N}_{2}\right]$ ratio measured in rocket-borne measurements is widely used to determine the turbopause altitude

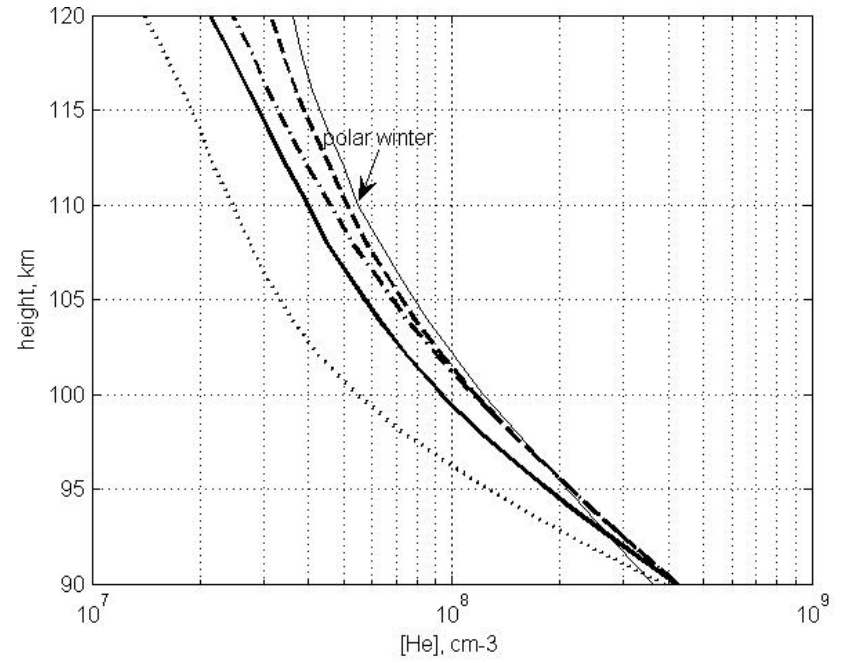

Fig. 3. $[\mathrm{He}]$ height profiles according to the MSIS-E-90 model: winter solstice at $40^{\circ} \mathrm{N}$ latitude at 14:00 LT (dashed curve), summer solstice at $40^{\circ} \mathrm{N}$ latitude at 14:00 LT (solid curve), the summer solstice at the equator at the same local time (dashed-dotted curve), and polar summer solstice at $70^{\circ} \mathrm{N}$ latitude (dotted curve), the longitude of $0^{\circ}$ for all data. All eddy diffusion coefficients are inferred from the MSIS-E-90 data, and the cooling rates corresponding to these coefficients are presented for the above given condition.

(von Zahn et al., 1990). An example of this ratio corresponding to the MSIS-E-90 data is shown in Fig. 2. In this case, the turbopause altitude of $90-92 \mathrm{~km}$ corresponds to the transition from an almost constant ratio to a significantly changed ratio.

Changes in the helium height distribution are largest in the transition region from the homosphere to the heterosphere. As seen from Fig. 3, these distributions differ at different latitudes and in different seasons. The steepest gradient of the [He] distribution corresponds to the largest eddy diffusion coefficient. This means that the eddy diffusion coefficient in summer should be larger than during winter at middle and high latitudes, and the maximum value of this coefficient should occur during polar summer. This qualitative estimate is in agreement with experimental turbulence data observed by a variety of techniques (see, for example, Hocking, 1986; Fukao et al., 1994; Lübken, 1997; Kelley et al., 2003).

The continuity equation for the chemical inert constituents is

$$
\begin{gathered}
\frac{\partial}{\partial z}\left[-D_{i}\left(\frac{\partial n}{\partial z}+\frac{n}{H_{\mathrm{He}}}+\left(1+\alpha_{T}\right) \frac{n}{T} \frac{\partial T}{\partial z}\right)\right] \\
+\frac{\partial}{\partial z}\left[-K_{\mathrm{ed}}\left(\frac{\partial n}{\partial z}+\frac{n}{H}+\frac{n}{T} \frac{\partial T}{\partial z}\right)\right]=0,
\end{gathered}
$$

where $n$ is the He density, $D_{i}$ and $K_{\text {ed }}$ are molecular and eddy diffusion coefficients, respectively, and $H$ and $H_{\mathrm{He}}$ are scale heights corresponding to the mean mass and the mass of helium, respectively. The molecular diffusion coefficient for the helium is determined by the formula 


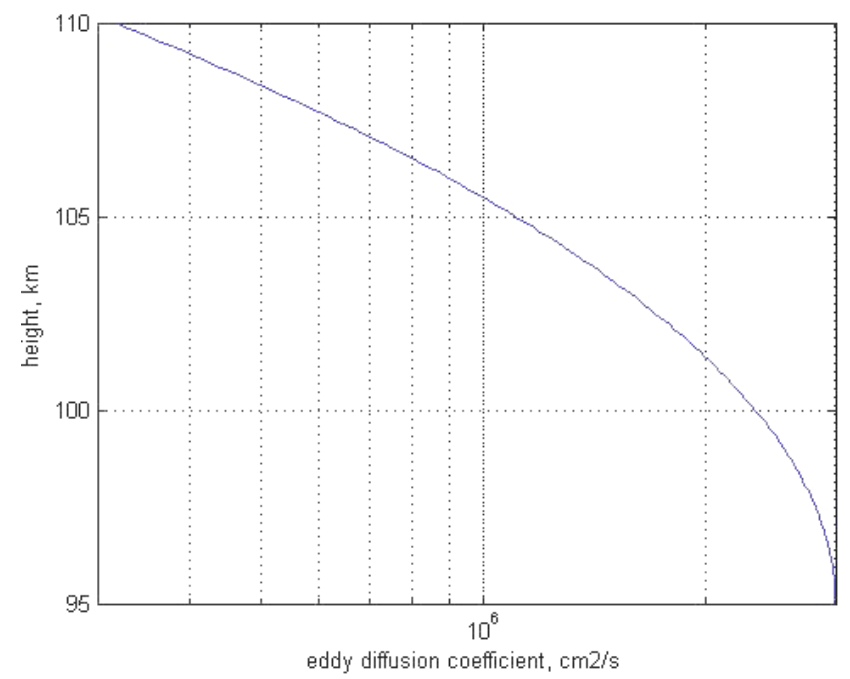

Fig. 4. Height profile of the eddy diffusion coefficient corresponding to the $[\mathrm{He}]$ height distribution shown by the dashed curve in Fig. 5a.

$D=\frac{\left(\sum_{i} A_{i} T^{s_{i}} n_{i} / N\right)}{N}$,

where $A_{i}$ and $s_{i}$ correspond to values for helium diffusion in $\mathrm{N}_{2}, \mathrm{O}_{2}$, and $\mathrm{O}$ given in Table 15.1 in Banks and Kockarts (1973), and $n_{i}$ and $N$ are the densities of these constituents and the total density, respectively. The altitude profile of $K_{\text {ed }}$ is given by the widely used approximation suggested by Shimazaki (1971):

$K_{\mathrm{ed}}=K_{\mathrm{ed}}^{m} \exp \left[-s\left(z-z_{m}\right)^{2}\right] \quad z>z_{m}$,

where $s$ is the reciprocal of the scale heights, $z_{m}$ is the height of the $K_{\text {ed }}$ peak, and $K_{\text {ed }}^{m}$ is the value of the peak. Note that Eq. (4) requires similar eddy and molecular diffusion, which means that the eddy scales must be much larger than the free pass of neutrals and much smaller than the scale height of the atmosphere.

Using the numerical solution of Eq. (4) and data on the densities and temperature given by the MSIS-E-90 model, it is possible to estimate the eddy diffusion coefficient in the region of the transition from the homosphere to the heterosphere. The [Ar]/[ $\left.\mathrm{N}_{2}\right]$ height profiles are also used to estimate the turbopause altitudes. The example of the height distribution of the eddy diffusion coefficient inferred using this approach is shown in Fig. 4.

Note that Eq. (4) does not include the term with vertical transport. The excellent agreement between the [He] height heterospheric profile at altitudes significantly higher than the turbopause altitude calculated by Eq. (4) and the [He] height profile given by the MSIS-E-90 model shows that the [He] distributions correspond exactly to the barometric law.
As seen from Fig. 5a, the [He] height profile calculated by Eq. (4) with the $K_{\text {ed }}$ profile shown in Fig. 4 is in good agreement with the [He] distribution given by the MSIS-E90 model. The same agreement exists in other cases (for example, see Fig. 5d), meaning that the eddy diffusion coefficients inferred using the [He] height distributions and the $[\mathrm{Ar}] /\left[\mathrm{N}_{2}\right]$ distributions are correct and can be used to further our study. The seasonal and latitudinal variations of the eddy diffusion coefficient estimated by this method are in good agreement with generally recognized variations, as seen from the $K_{\text {ed }}$ values given in the captions to Fig. 5a, b, and d. Also, these variations coincide with the qualitative estimate corresponding to the $[\mathrm{He}]$ variations, as mentioned in the second paragraph of this section.

\section{Heating and cooling induced by eddy turbulence corresponding to the eddy diffusion coefficients inferred from MSIS-E-90 data}

The eddy turbulence heating/cooling rate is given by equation (Vlasov and Kelley, 2010)

$$
\begin{aligned}
Q_{\mathrm{ed}}= & \frac{\partial}{\partial z}\left[K_{\mathrm{eh}} C_{p} \rho\left(\frac{\partial T}{\partial z}+\frac{g}{C_{p}}\right)\right] \\
& +K_{\mathrm{eh}} \rho \frac{g}{T c}\left(\frac{\partial T}{\partial z}+\frac{g}{C_{p}}\right),
\end{aligned}
$$

where $K_{\text {eh }}$ is the coefficient of eddy heat transport, $\rho$ is the undisturbed gas density, $g$ is the gravitational acceleration, $T$ is the temperature, $C_{p}$ is the specific heat at constant pressure, and $c$ is a dimensionless constant commonly taken to be 0.8. The vertical energy flux is given by the formula (A07)

$F_{\Theta}=-\rho C_{p} \Pi K_{\mathrm{ec}} \frac{\partial \Theta}{\partial z}$

or the formula (BM09)

$F_{\Theta}=-K_{\text {eh }} \frac{\partial \Theta}{\partial z}$,

where $\Theta=T / \Pi$ is the potential temperature. As seen from the formula given below, the flux given by Akmaev is the same as the flux in the eddy heating rate term in Eq. (6),

$F_{\Theta}=-\rho C_{p} \Pi K_{\mathrm{ec}} \frac{\partial \Theta}{\partial z}=-\rho C_{p} K_{\mathrm{eh}}\left(\frac{\partial T}{\partial z}+\frac{g}{C_{p}}\right)$,

which corresponds to the flux in the fourth term on the righthand side of Eq. (11) in BM09 and also corresponds to the flux in the $\mu$ term without molecular diffusion but with an "eddy diffusion coefficient from the GW parameterization and vertical diffusion scheme". The first term in Eq. (10) in BM09 can be presented as $K_{\mathrm{eh}} \omega_{b}^{2}$ because

$\varpi_{\mathrm{B}}^{2}=g\left(\frac{1}{\Theta} \frac{\partial \Theta}{\partial z}\right)$. 


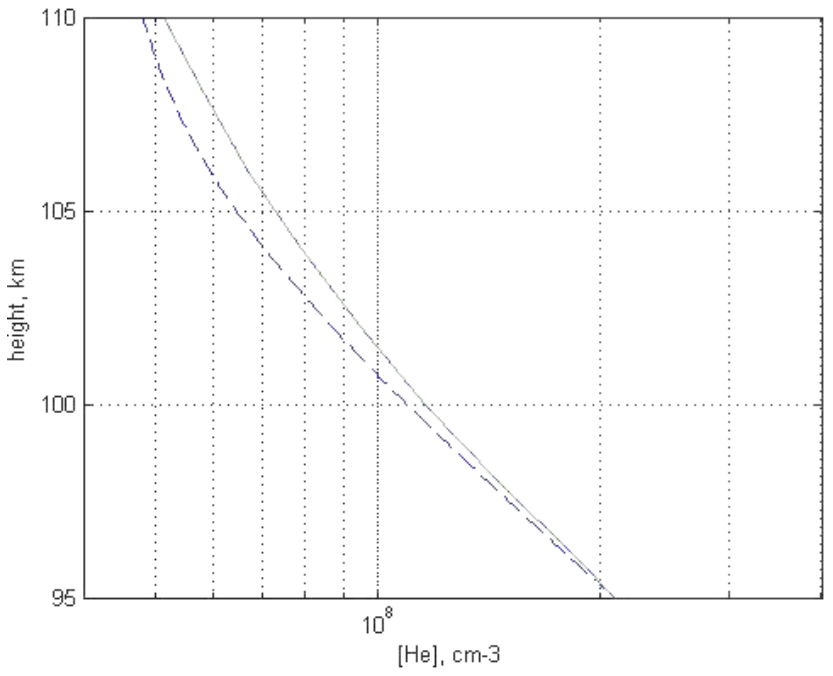

Fig. 5a. $[\mathrm{He}]$ height profiles in winter solstice given by the MSISE-90 model (solid curve) and calculated (dashed curve) with the eddy diffusion coefficient shown in Fig. $4\left(K_{\text {ed }}^{m}=3 \times 10^{6} \mathrm{~cm}^{2} \mathrm{~s}^{-1}\right.$, $z_{m}=95 \mathrm{~km}, s=0.01 \mathrm{~km}^{-2}$ ).

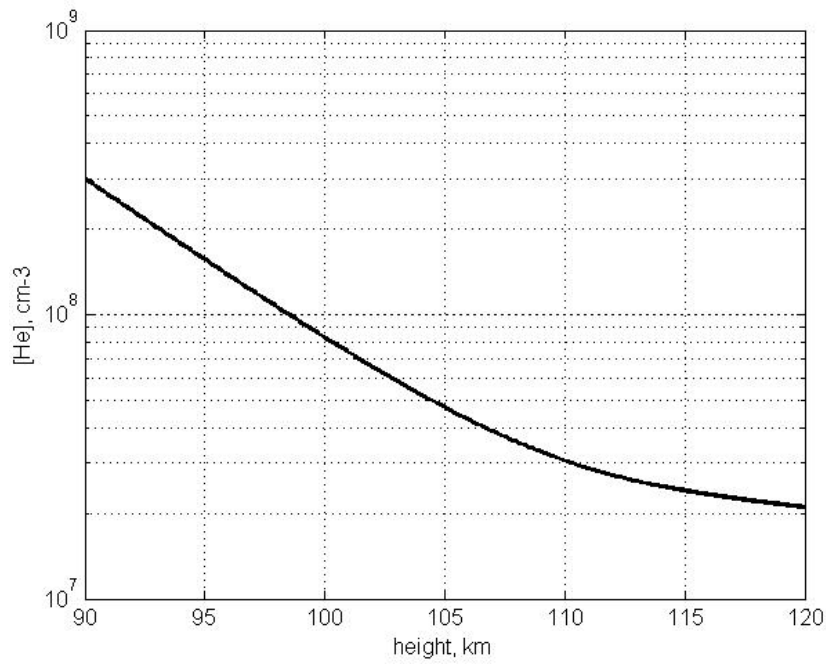

Fig. 5b. The same as in Fig. 5a but in summer solstice with $K_{\text {ed }}^{m}=$ $6 \times 10^{6} \mathrm{~cm}^{2} \mathrm{~s}^{-1}, z_{m}=92 \mathrm{~km}$, and $s=0.01 \mathrm{~km}^{-2}$.

This term is similar to the second term in our Eq. (6), but our term includes the coefficient $c$ determined by the ratio of the Richardson number, $R i=\varpi_{\mathrm{B}}^{2} /(\partial u / \partial z)^{2}$, to the turbulent Prandtl number, $P=K_{\mathrm{em}} / K_{\mathrm{eh}}$, for the steady mean motion ( $K_{\mathrm{em}}$ is the eddy momentum transport coefficient). Changing the $c$ value, we take into account the effect of gravity waves. Thus, the second term in our Eq. (6) includes the first term of Eq. (10) and the $\varepsilon_{m}$ term in Eq. (11) (BM09). Excluding the $\varepsilon_{\mathrm{GW}}$ term, we see that our Eq. (6) accumulates the terms of Eq. (11) but with different eddy diffusion coefficients. Thus, the formulas for the eddy heating/cooling rate coincide with formulas for these rates that were obtained and

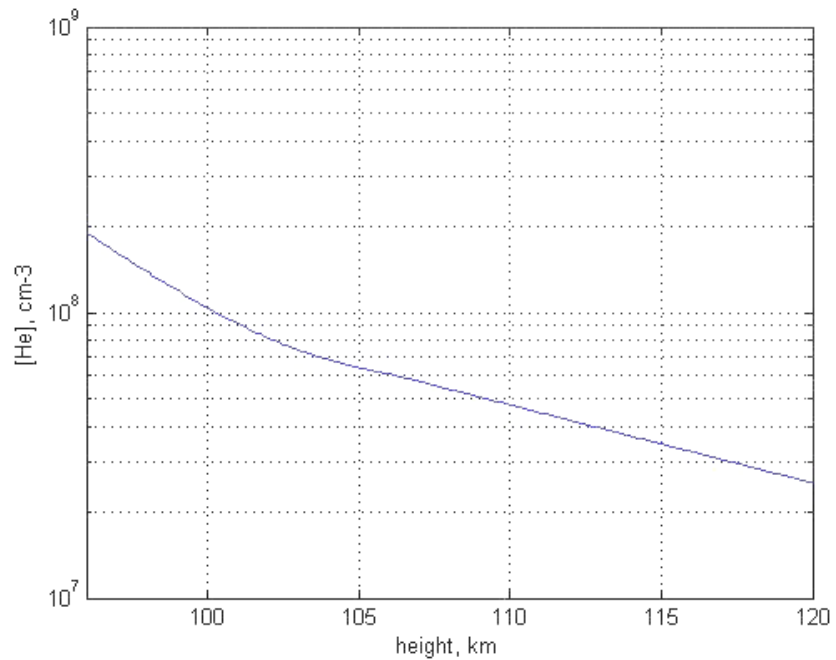

Fig. 5c. The same as in Fig. 5a but at the equator.

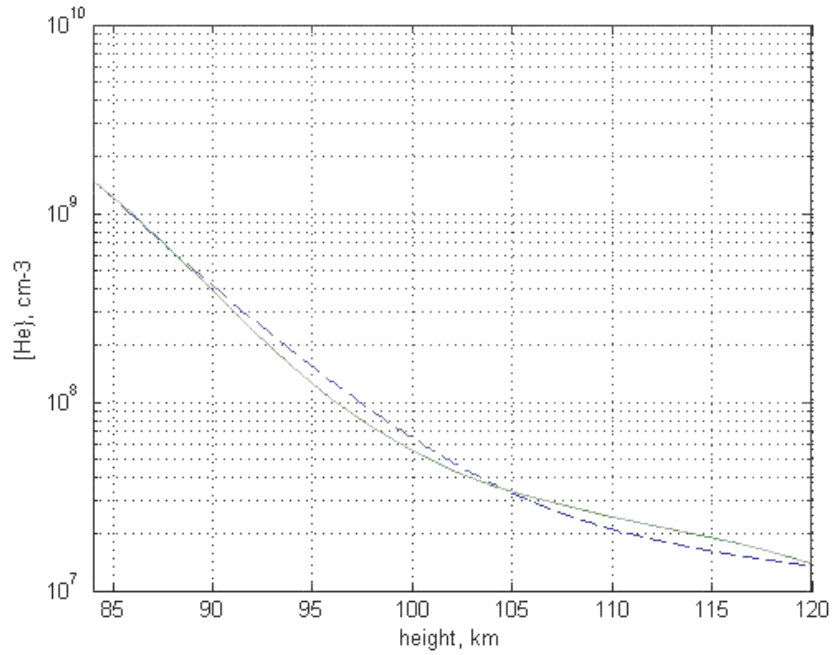

Fig. 5d. $[\mathrm{He}]$ height profile given by the MSIS-E-90 model in the polar region (solid line) and calculated by Eq. (4) with $K_{\text {ed }}^{m}=7 \times$ $10^{6} \mathrm{~cm}^{2} \mathrm{~s}^{-1}, z_{m}=84 \mathrm{~km}$, and $s=0.01 \mathrm{~km}^{-2}$ (dashed curve).

used in estimating the thermal effect of gravity waves (A07, BM09). This means that heating/cooling by gravity waves can be described by eddy turbulence heat transport. The discussion above shows that the impact of gravity waves on thermal balance can be described by eddy heat transport.

The cooling rates calculated with $K_{\text {ed }}=K_{\text {eh }}$ and shown in Fig. 6a-d are larger by a factor of 2 or 3 than the normal cooling rates corresponding to the temperature given by the MSIS-E-90 model. This means that the eddy heat transport coefficient should be less than the eddy diffusion coefficient. 


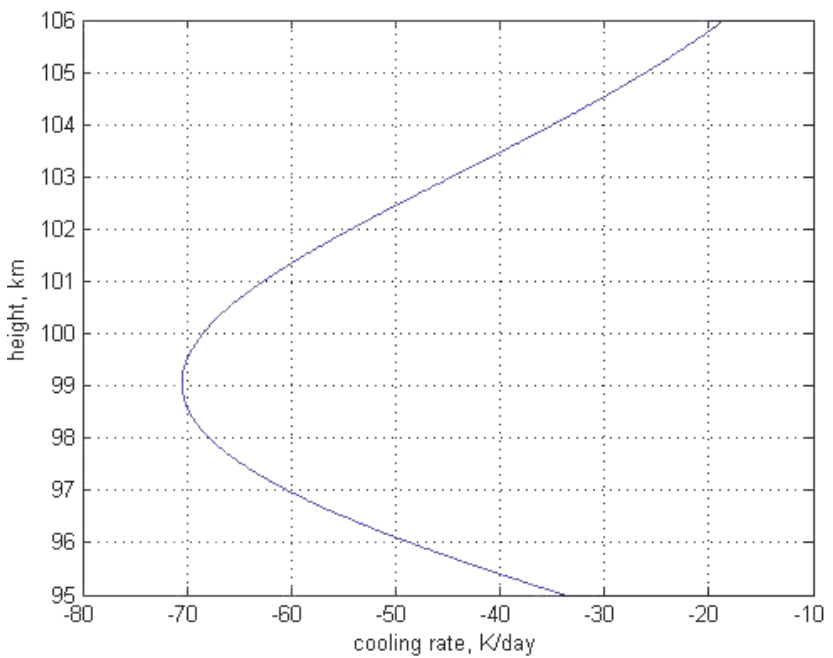

Fig. 6a. Height profile of the cooling rate in winter solstice calculated with the coefficient of eddy heat transport equaled to the eddy diffusion coefficient shown in Fig. 2.

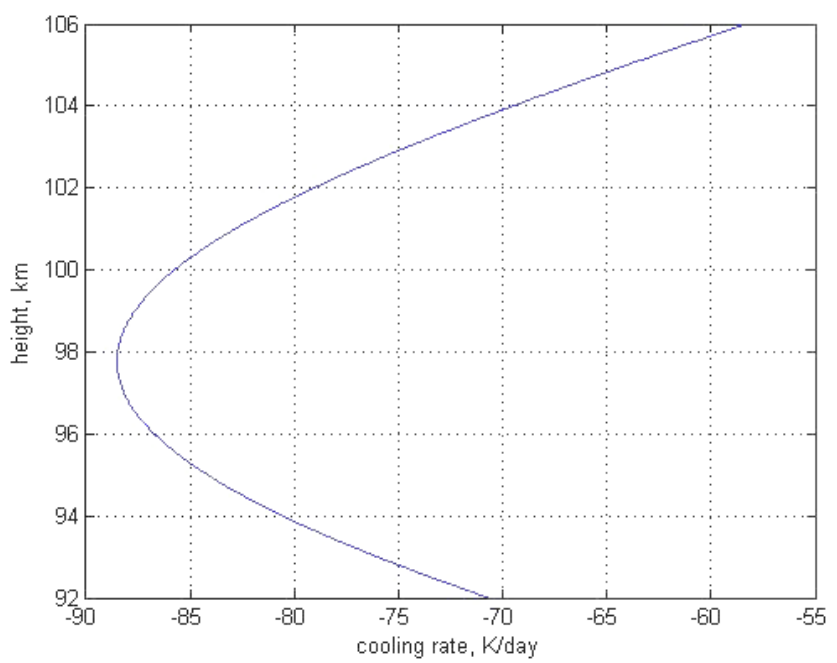

Fig. 6b. Height profiles of the cooling rate calculated with $K_{\mathrm{eh}}=$ $K_{\text {ed }}$ inferred from the $[\mathrm{He}]$ distribution in summer solstice $\left(K_{\mathrm{ed}}^{m}=\right.$ $\left.5 \times 10^{6} \mathrm{~cm}^{2} \mathrm{~s}^{-1}, s=0.01 \mathrm{~km}^{-2}, c=0.8, P=1\right)$.

\section{Uniform, localized, and large-scale turbulence}

Uniform turbulence is characterized by a wide spectrum of eddy scales, from scales much larger than the free path to scales much smaller than the atmospheric scale height. In this case, the eddy momentum and heat transport coefficients are equal and the Prandtl number $P$ equals 1 . However, more complex turbulence can take place. Observations of artificial clouds and meteor trails show that turbulence is very intermittent, both temporarily and spatially (Kelley et al., 2003; Bishop et al., 2004). The thin turbulent layers separated by regions of very weak turbulence or laminar flow occur. An ensemble of gravity waves can produce turbulent layers with

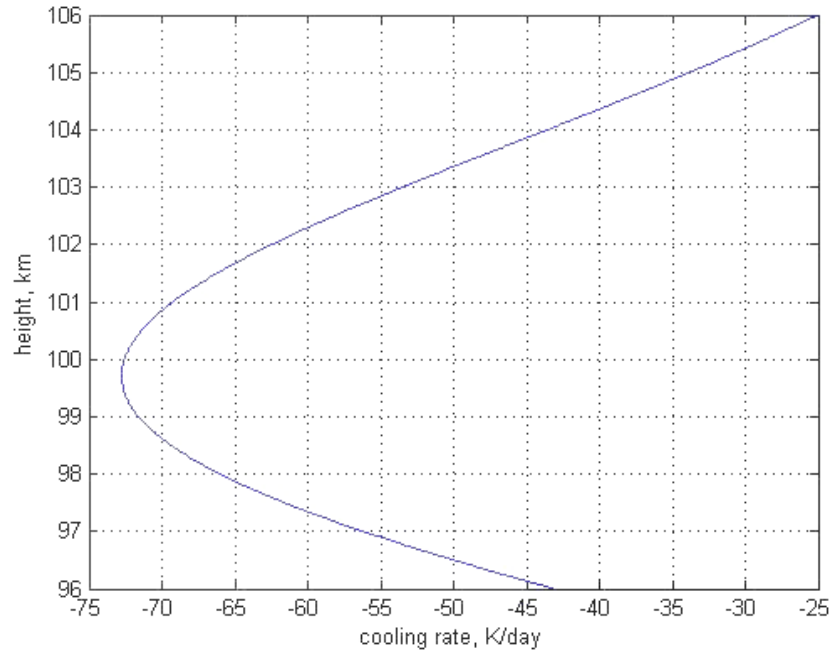

Fig. 6c. Height profile of the cooling rate calculated with $K_{\mathrm{eh}}=$ $K_{\text {ed }}$ inferred from the [He] distribution at the summer equator $\left(K_{m}=3 \times 10^{6} \mathrm{~cm}^{2} \mathrm{~s}^{-1}, s=0.02 \mathrm{~km}^{-2}, R i=0.8\right)$.

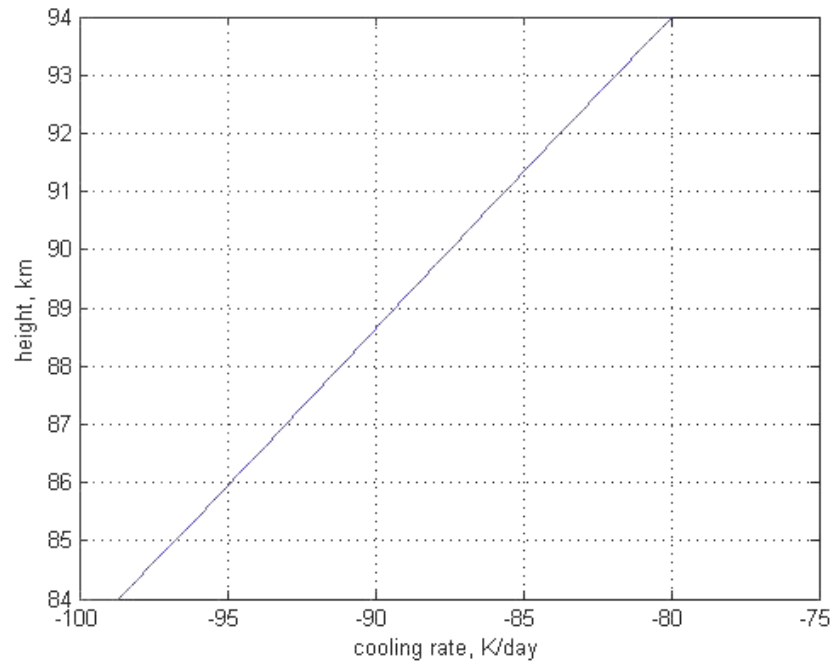

Fig. 6d. Height profiles of the cooling rate calculated with $K_{\mathrm{eh}}=$ $K_{\text {ed }}$ inferred from the [He] distribution at polar summer solstice $\left(K_{\text {ed }}^{m}=5 \times 10^{6} \mathrm{~cm}^{2} \mathrm{~s}^{-1}, s=0.002 \mathrm{~km}^{-2}, c=0.4, P=2, z_{m}=\right.$ $84 \mathrm{~km})$.

a thickness of a few tens of meters to a kilometer. This problem was discussed by Hocking (1999). Observations of artificial clouds and meteor trails show that turbulence is very intermittent, both temporarily and spatially (Kelley et al., 2003; Bishop et al., 2004). In this case, the average eddy diffusion and eddy heat transport coefficients can differ, and the Prandtl number can exceed 1 and may be up to 3 (Fritts and Dunkerton, 1985). The Prandtl number is determined by the ratio

$P=C_{p} \mu / \lambda$, 


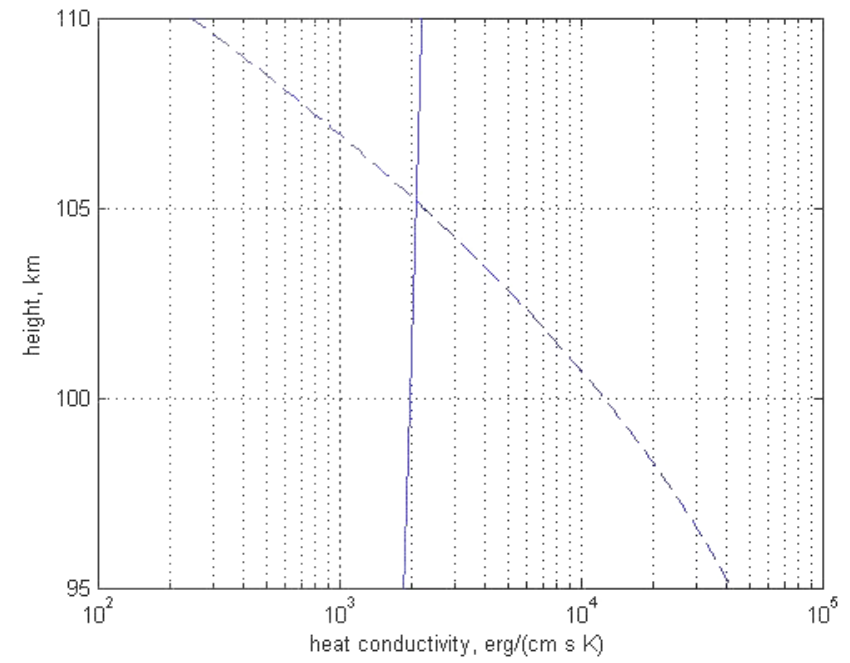

Fig. 7a. Height distributions of the thermal conductivity (solid curve) and eddy heat conductivity (dashed curve) with the eddy heat transport coefficient equaled to $K_{\text {ed }}$, shown in Fig. 4. Eddy heat conductivity is given by the formula $\lambda_{\mathrm{ed}}=K_{\mathrm{ec}} C_{p} \rho \operatorname{erg}(\mathrm{cm} \mathrm{s} \mathrm{K})^{-1}$, and the thermal heat conductivity is given by the formula $\lambda_{\text {th }}=38.2 T^{0.69}+1.9 T+51.4 \mathrm{erg}(\mathrm{cm} \mathrm{s} \mathrm{K})^{-1}$ (Banks and Kockarts, 1973).

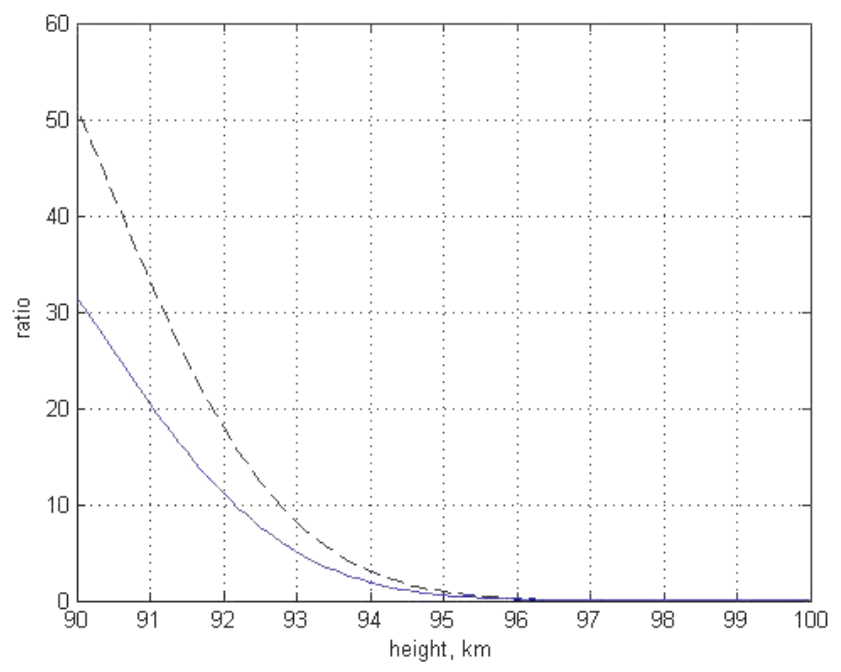

Fig. 7b. Ratio of the eddy diffusion and molecular diffusion coefficients (dashed curve) and the ratio of eddy heat conductivity to the thermal conductivity (solid curve) calculated with the eddy diffusion coefficient given by L97 for polar summer.

where $\mu$ is the dynamic viscosity and $\lambda$ is the thermal conductivity. Using the values of these parameters $\quad\left(\mu=3.8 \times 10^{-6} T^{0.69} \mathrm{~g} \mathrm{~cm}^{-1} \mathrm{~s}^{-1}, \quad \lambda=\right.$ $\left.38.2 \times T^{0.69}+1.9 T+51.4 \mathrm{erg} \mathrm{cm}^{-1} \mathrm{~s}^{-1} \mathrm{~K}^{-1}\right)$ given by Banks and Kockarts (1973), $P=0.47$ can be found for $\mathrm{N}_{2}$ and $T=200 \mathrm{~K}$. The relation

$P=K_{\mathrm{ed}} / K_{\mathrm{eh}}$

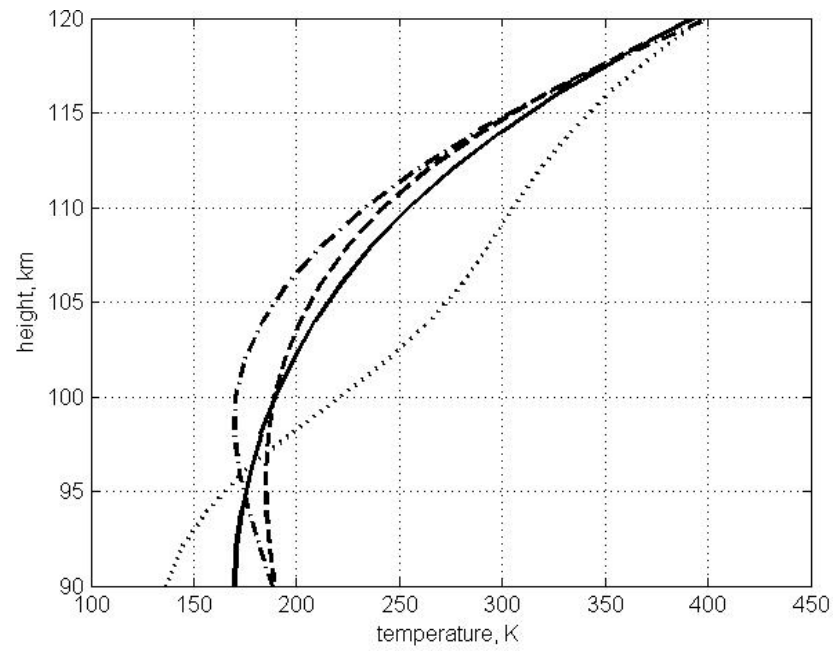

Fig. 8. Temperature height profiles in winter (dashed curve) and summer solstices (solid curve) at middle latitudes, at the equator (dashed-dotted curve), and at high latitudes (dotted curve), as given by the MSIS-E-90 model.

can be obtained for eddy turbulence because, in this case, $\mu \propto K_{\text {ed }} \rho$ and $\lambda \propto K_{\mathrm{eh}} \rho C_{p}$. Obviously, eddy turbulence increases the viscosity. If we assume that the gravity wave energy dissipates to wind shear in the turbopause region, we could use the formula $K_{\mathrm{em}}=0.81 \varepsilon / \omega_{\mathrm{B}}^{2}$ (Fritts and Luo, 1995). This formula is the same as the formula used for $K_{\text {ed }}$ (Fritts and Luo, 1995; Lübek, 1997), and Eq. (11) can be applied because $P=K_{\text {em }} / K_{\text {eh }}$. As seen from Fig. 7a, the eddy heat conductivity can be much higher than the molecular thermal conductivity in spite of the large Prandtl number. The ratios of the eddy diffusion coefficient to the molecular diffusion coefficient and the eddy heat conductivity to the thermal conductivity are large at the $K_{\text {ed }}$ peak altitude, as can be seen from Fig. $7 b$.

Analysis of Eq. (6) shows that the negative vertical gradient of the eddy heat transport coefficient is the main cause of the cooling. This gradient is located in the turbopause region, and the turbopause altitude depends on this gradient.

Note that, according to the MSIS-E-90 data, conditions for developing Kelvin-Helmholtz instabilities in the turbopause region do not exist because the Richardson number is larger than 0.25 and the temperature gradient is positive or close to zero (see Fig. 8). However, these data presented average conditions. In the real atmosphere, there are significant fluctuations corresponding to turbulence that provide mixing of the atmospheric constituents.

Localized turbulence consists of unstable layers with a thickness $L$ of about $100-1000 \mathrm{~m}$ and intermediate regions with molecular diffusion and thermal conductivity. It is possible to assume that these thin layers are limited by boundaries similar to those in the pipe. The wind shear maximum is at $100 \mathrm{~km}$ (Larsen, 2002). The averaged value of the wind velocity at $100 \mathrm{~km}$ is about $50 \mathrm{~m} \mathrm{~s}^{-1}$. The Reynolds number 


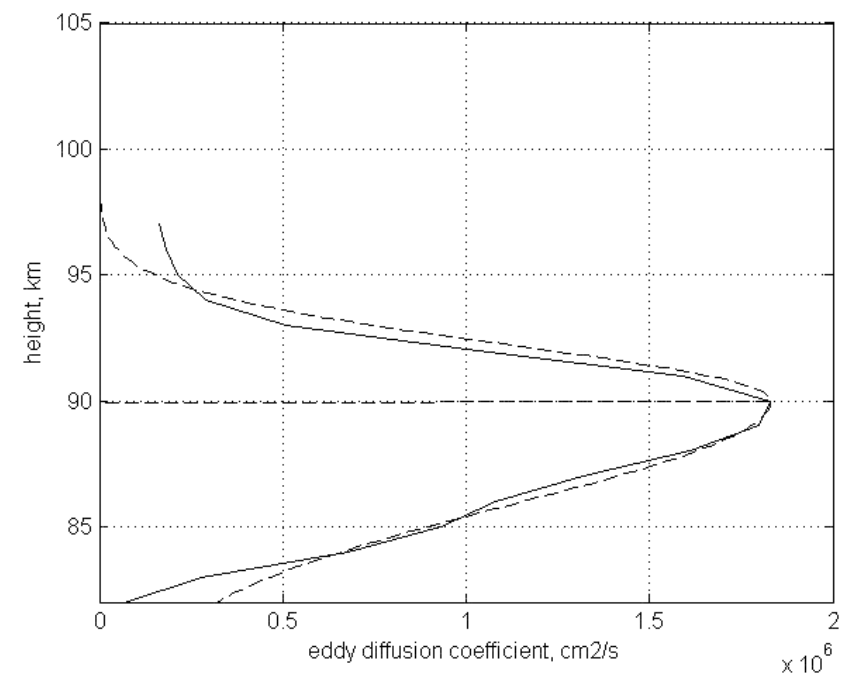

Fig. 9. Height profile of the eddy diffusion coefficient measured by Lübken (1997) in the polar region (solid curve) and approximated by Eq. (5) (dashed curve) with parameters $K_{\text {ed }}^{m}=1.83 \times$ $10^{6} \mathrm{~cm}^{2} \mathrm{~s}^{-1}$ and $z_{m}=90 \mathrm{~km}$, shown by the horizontal line.

is given by the relation

$\operatorname{Re}=\rho U L / \mu$,

where $U$ is the velocity, $L$ is the characteristic pipe diameter, and $\mu=3.8 \times 10^{-6} T^{0.69} \mathrm{~g} \mathrm{~cm}^{-1} \mathrm{~s}^{-1}$ is the dynamic viscosity for the mixture of $\mathrm{N}_{2}$ and $\mathrm{O}_{2}$ (Bank and Kockarts, 1973). For example, using density $\rho=6 \times 10^{-10} \mathrm{~g} \mathrm{~cm}^{-3}$ and temperature $T=180 \mathrm{~K}$ at $100 \mathrm{~km}$ as given by the MSIS-E-90 model, the velocity $\rho=6 \times 10^{-10} \mathrm{~g} \mathrm{~cm}^{-3}$ and temperature $T=180 \mathrm{~K}$ at $100 \mathrm{~km}$ given by the MSIS-E-90 model, and the velocity $U=50 \mathrm{~m} \mathrm{~s}^{-1}$ corresponding to $U$ for the mean wind at $100 \mathrm{~km}$ with $L=1 \mathrm{~km}$, a Reynolds number of 2300 can be found. Turbulence can develop when the Reynolds number exceeds 1000 . In this case, localized instabilities can occur. However, a problem exists with the thin turbulent layer boundary conditions, and applying this approach to free atmospheric gas may be a questionable assumption. Note that low Richardson numbers of $R i<0.25$ are possible in the narrow range of altitudes around $100 \mathrm{~km}$ where strong wind shear occurs, according to Larsen (2002). However, these low numbers simultaneously need the low $\omega_{\mathrm{B}}$ value that large negative temperature gradients can provide. According to MSIS-E-90 data, however, the temperature gradient is very small in the turbopause region.

Let us compare the cooling rate calculated with the eddy diffusion coefficient estimated by Lübken (1997, hereafter referred to as L97) and shown in Fig. 10 and the cooling rate shown in Fig. 6d. Both rates correspond to the polar summer. As seen from the cooling rates shown in Figs. $6 \mathrm{~d}$ and 10, the maximum cooling rate corresponding to the $K_{\text {ed }}$ of L97 is larger than the maximum rate shown in Fig. 6d, but the $K_{\text {ed }}^{m}$ values of the latter are less by a factor of 2.5 than the $K_{\text {ed }}^{m}$

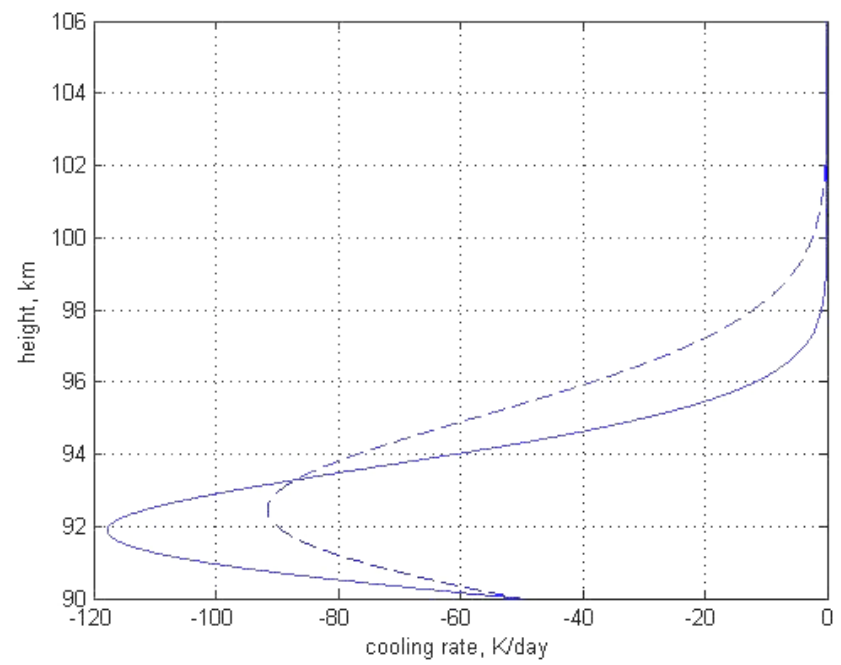

Fig. 10. Height profiles of the cooling rate (solid curve) calculated with the eddy diffusion coefficient shown by a dashed curve $\left(s=0.1 \mathrm{~km}^{-2}\right)$ in Fig. 9 and calculated with $s=0.03 \mathrm{~km}^{-2}$ corresponding to agreement with the cooling rate induced by the eddy diffusion coefficient shown in Fig. 9 below the $K_{\text {ed }}$ peak. The straight line shows this peak.

of L97. This result shows a very important role of the $K_{\text {ed }}$ gradient above the peak characterized by the $s$ value, which is much larger for the $K_{\text {ed }}$ profile given by L97 (see Fig. 9).

The very important role of the $K_{\text {ed }}$ gradient above the peak can be seen by analyzing the thermal equation. This equation can be written as

$$
\begin{aligned}
& K_{\mathrm{eh}} C_{p} \frac{\partial^{2} T}{\partial z^{2}}+C_{p}\left(\frac{\partial K_{\mathrm{eh}}}{\partial z}-\frac{K_{\mathrm{eh}}}{H}\right) \frac{\partial T}{\partial z}+g\left(\frac{\partial K_{\mathrm{eh}}}{\partial z}-\frac{K_{\mathrm{eh}}}{H}\right) \\
& \quad+\varepsilon+q-L=0
\end{aligned}
$$

which includes $Q_{\text {eh }}$ given by Eq. (6), heating due to the energy dissipation of gravity waves, $\varepsilon$, chemical heating and heating by the ultraviolet solar radiation, $q$, and cooling by the $\mathrm{CO}_{2}$ and $\mathrm{O}$ infrared radiation, $L$. According to the conditions (L97), the $K_{\text {ed }}$ peak is in the mesopause and Eq. (13) can be simplified to the relation

$K_{\mathrm{eh}}^{m} g / H=q+\varepsilon-L$.

Using this relation with the $\varepsilon$ value given in Table 3 in Lübken (1997) at the $K_{\text {ed }}$ peak altitude and $(q-L) \leq$ $10 \mathrm{~K} \mathrm{day}^{-1}$, the $K_{\mathrm{eh}}^{m}$ value can be found to be $\leq 1.1 \times$ $10^{6} \mathrm{~cm}^{2} \mathrm{~s}^{-1}$. This value is significantly less than $K_{\mathrm{eh}}^{m}=$ $1.83 \times 10^{6} \mathrm{~cm}^{2} \mathrm{~s}^{-1}$ as estimated by L97, but the maximum cooling with this lower value is $-45 \mathrm{~K} \mathrm{day}^{-1}$ due to the large value of $s$ corresponding to the $K_{\text {ed }}$ height profile given by L97. The $s$ value should decrease by a factor of 10 to achieve thermal balance at altitudes above the $K_{\text {ed }}$ peak. Note that the cooling peak occurs above the $K_{\text {ed }}$ peak altitude. In any case, the cooling induced by the eddy diffusion measured by 


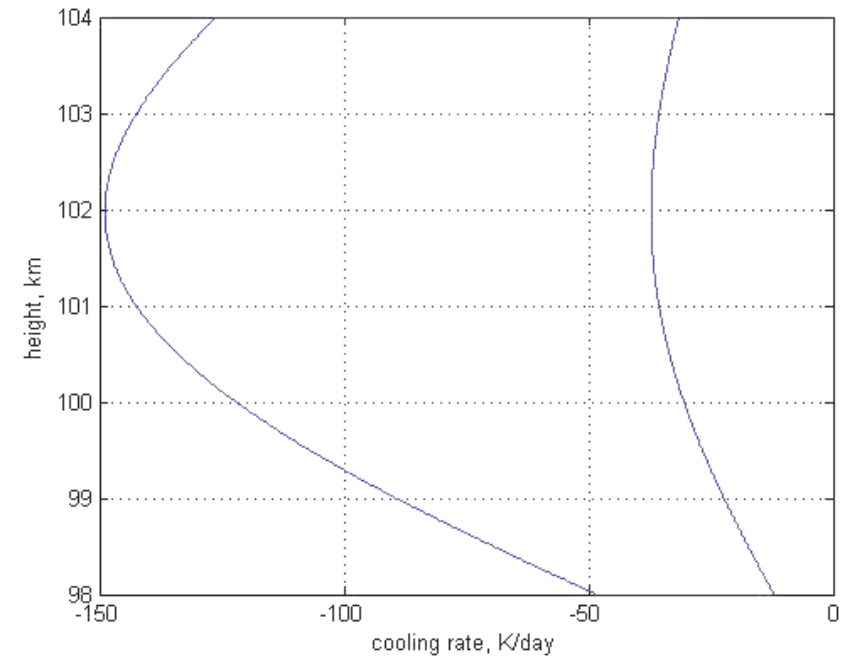

Fig. 11. Cooling rates calculated with the eddy diffusion shown in Fig. 3 in Hecht et al. (2004) (curve with the peak value of $-37 \mathrm{Kday}^{-1}$ ) and with the eddy diffusion coefficient with $K_{\mathrm{ed}}^{m}=$ $1 \times 10^{7} \mathrm{~cm}^{2} \mathrm{~s}^{-1}$ (curve with the peak value of $-150 \mathrm{~K} \mathrm{day}^{-1}$ ) corresponding to the values given in Table 2 in Bishop et al. (2004).

Lübken is very large. In this case, the $P$ value was larger than 2 , indicating localized turbulence.

Following L97 and using the formulas given by Weinstock $(1978,1981)$, we obtain the formula

$L_{\mathrm{ed}}=10.46 K_{\mathrm{eh}}^{3 / 4} \varepsilon^{-1 / 4}$,

where $L_{\mathrm{ed}}$ is the outer turbulence scale and $\varepsilon$ is the turbulent energy dissipation rate. Using the $\varepsilon$ values measured by L97 during the summer and the $K_{\text {ed }}$ profiles given in Table 3 in L97, it is possible to estimate $L_{\mathrm{ed}}=0.97 \mathrm{~km}$ in the $K_{\text {ed }}$ peak. This turbulence scale is comparable to the scale height of atmospheric gas and is an indication of localized turbulence.

The introduction mentioned that very high eddy diffusion coefficients were estimated in TOMEX using the energy deposition rates inferred from the observed passive tracer trails (Bishop et al., 2004). Also, the eddy diffusion coefficients were inferred from airglow observed during TOMEX (Hecht et al., 2004). The latter were less by a factor of 5-10 than the former. We could not find an explanation for this huge disagreement. The cooling rates calculated by Eq. (6) with the $K_{\text {ed }}$ height profile shown in Fig. 3 in Hecht et al. (2004) and the same profile but normalized by $K_{\text {ed }}^{m}=1 \times 10^{7} \mathrm{~cm}^{2} \mathrm{~s}^{-1}$ are shown in Fig. 11. The cooling rate calculated with the low eddy diffusion coefficient corresponds to the normal cooling rates, but the cooling rate with a high eddy diffusion coefficient is very high and we must assume that the Prandtl number is 3 in this case. We assume that the rate coefficients inferred from a chemical release by Bishop et al. (2004) are overestimated.

In the introduction, we also mentioned the very large energy deposition rate of $\varepsilon=2 \mathrm{~W} \mathrm{~kg}^{-1}=200 \mathrm{Kday}^{-1}$, estimated by Szewczyk et al. (2013) based on the results of their rocket experiment to measure density fluctuations. The maximum value of the eddy diffusion coefficient for the peak of the smoothed energy dissipation rate shown in Fig. 1b (green curve) in Szewczyk et al. (2013) and the $\omega_{\mathrm{B}}^{2}$ value corresponding to the temperature height profile within the altitude range of 89-92 km shown in Fig. 1a (black curve) in Szewczyk et al. (2013) can be found to be $7.5 \times 10^{7} \mathrm{~cm}^{2} \mathrm{~s}^{-1}$. This $K_{\text {ed }}^{m}$ value is higher by a factor of about 3 than the value estimated by Bishop et al. (2004). In this case, the cooling rates above the $K_{\text {ed }}^{m}$ peak are much higher than the cooling rates calculated for the $K_{\text {ed }}^{m}$ peak given by Bishop et al. (2004) and shown in Fig. 11. Assuming a large Prandtl number, it is possible to decrease the $K_{\text {eh }}$ value and cooling rate. However, the $K_{\text {ed }}$ value is very high, and the impact of eddy diffusion on neutral composition is very strong. For example, very low atomic density can be found (Vlasov and Kelley, 2010), contradicting all of the experimental data generalized by the MSIS-E-90 model. Taking this effect into account, this very high energy deposition rate can be assumed to occur for $1 \mathrm{~h}$ but no longer. Note that the observed inversion layers (double mesopause) are usually characterized by small gradients and the temperature enhancements (Yu and She, 1995). A heating rate of about $30-40 \mathrm{~K} \mathrm{day}^{-1}$ can provide these temperature enhancements (Vlasov and Kelley, 2012). For example, note that the normal heating rate at $90 \mathrm{~km}$ does not exceed $10 \mathrm{~K} \mathrm{day}^{-1}$ (Roble, 1995).

We plan to analyze methods for estimating eddy diffusion coefficients, based on density fluctuation measurements and passive tracer trails, in the near feature.

\section{Conclusions}

Using the continuity equation and the $[\mathrm{He}],[\mathrm{Ar}]$, and $\left[\mathrm{N}_{2}\right]$ height distributions and other parameters given by the MSISE-90 model, the eddy diffusion coefficients are estimated for the turbopause region. The latitudinal and seasonal variations of the eddy diffusion coefficients inferred from the chemical inert constituent distributions given by the MSIS-E-90 model are in good agreement with observations of these variations.

The cooling rates calculated with the eddy heat transport coefficients equaled to the eddy diffusion coefficients are found to be much larger than the normal cooling rates corresponding to temperatures given by the MSIS-E-90 model. In this case, the eddy heat transport coefficient should decrease by a factor of 2 and above, and the Prandtl number may be 2 or larger. Also, very strong cooling is estimated for the eddy diffusion coefficients inferred from the experimental data, meaning that localized turbulence should exist in the turbopause region.

According to these results, eddy turbulence always cools atmospheric gas in the turbopause region. This cooling strongly depends on the eddy diffusion coefficient gradient above the $K_{\text {ed }}$ peak. However, it is impossible to significantly 
decrease this gradient because, in this case, the turbopause altitude can be found at altitudes above $110-115 \mathrm{~km}$, contradicting the MSIS-E-90 model and other experimental data.

There are no conditions for developing dynamic or convective instability in the turbopause region because of the high buoyancy frequency value $\omega_{\mathrm{B}}^{2} \approx 4.5 \times 10^{-4} \mathrm{~s}^{-1}$. We consider the average condition corresponding to the MSIS-E-90 model, which means that the average value of the wind shear can be used. The value does not exceed $20 \mathrm{~m}^{-1} \mathrm{~s}^{-1} \mathrm{~km}^{-1}$ (Larsen, 2002), and the $R i$ value is about 1 . We suggest that the criterion for developing localized turbulence may differ from the usual criterion and that localized turbulence may develop as a result of strong wind with a mean wind shear in the narrow layers of turbulence, limited by boundaries with undisturbed gas. In this case, the gas transport inside a turbulent layer may be compared to flow in a narrow pipe. In any case, the development of localized turbulence in the turbopause region needs further study.

The existence of localized turbulence in the turbopause region is important in understanding the effect of eddy turbulence on thermal balance in the MLT. We conclude that a new approach for the parameterization of energy deposition by gravity waves is needed. It must be emphasized that the localized turbulence is found by using the data given by the MSIS-E-90 model corresponding to the averaged conditions. Thus, our knowledge of the mean thermal structure in the transition region from the homosphere to the heterosphere will improve due to a better understanding of the specific features of mean dynamic forcing.

Acknowledgements. Work at Cornell was supported by the National Science Foundation under grant ATM-0551107.

Topical Editor C. Jacobi thanks two anonymous referees for their help in evaluating this paper.

\section{References}

Akmaev, R. A.: On the energetics of mean-flow interactions with thermally dissipating gravity waves, J. Geophys. Res., 112, D11125, doi:10.1029/2006JD007908, 2007.

Banks, P. M. and Kockarts, G.: Aeronomy, part B, Academic Press, New York, 11-12, 1973.

Becker, E.: Direct heating rates associated with gravity wave saturation, J. Atmos. Sol.-Terr., 66, 683-696, 2004.

Becker, E. and McLandress, C.: Consistent scale interaction of gravity waves in the Doppler spread parameterization, J. Atmos. Sci., 66, 1434-1449, 2009.

Bishop, R. L., Larsen, M. F., Hecht, J. H., Liu, A. Z., and Gardner, C. S.: TOMEX: Mesospheric and lower thermospheric diffusivity and instability layers, J. Geophys. Res., 109, D02S03, doi:10.1029/2002JD003079, 2004.

Blum, P. W. and Schuchardt, K. G. H.: Semi-theoretical global models of the eddy diffusion coefficient based on satellite data, J. Atmos. Terr. Phys., 40, 1137-1142, 1978.
Das, U., Sinha, H. S. S., Sharma, S., Chandra, H., and Das, S. K.: Fine structure of the low-latitude mesospheric turbulence, J. Geophys. Res., 114, D10111, doi:10.1029/2008JD011307, 2009.

Fritts, D. C.: Gravity wave saturation in the middle atmosphere: A review of theory and observations, Rev. Geophys., 82, 275-308, 1984.

Fritts, D. C. and Alexander, M. J.: Gravity wave dynamics and effects in the middle atmosphere, Rev. Geophys., 41, 1003, doi:10.1029/2001RG000106, 2003.

Fritts, D. C. and Dunkerton, T. J.: Fluxes of heat and constituents due to convective unstable gravity waves, J. Amos. Sci., 42, 549556, 1985.

Fritts, D. C. and Luo, Z.: Dynamical and radiative forcing of the summer mesopause circulation and thermal structure, J. Geophys. Res., 100, 3119-3128, 1995.

Fukao, S., Yamanaka, M. D., Ao, N., Hocking, W. K., Sato, T., Yamamoto, M., Nakamura, T., Tsuda, T., and Kato, S.: Seasonal variability of vertical eddy diffusivity in the middle atmosphere, 1. Three-year observations by the middle and upper atmosphere radar, J. Geophys. Res., 99, 18973-18987, 1994.

Gardner, C. S., Zhao, Y., and Liu, A. Z.: Atmospheric stability and gravity wave dissipation in the mesopause region, J. Atmos. Sol.Terr., 64, 923-929, 2002.

Gordiets, B. F., Kulikov, Yu. N., Markov, M. N., and Marov, M. Ya.: Numerical modeling of the thermospheric heat budget, J. Geophys. Res., 87, 4504-4514, 1982.

Hecht, J. H., Liu, A. Z., Walterscheid, R. L., Roble, R. G., Larsen, M. F., and Clemmons, J. H.: Airglow emissions and oxygen mixing ratios from the photometer experiment on the Turbulent Oxygen Mixing Experiment (TOMEX), J. Geophys. Res., 109, D02S05, doi:10.1029/2002JD003035, 2004.

Hedin, A. E.: Extension of the MSIS thermosphere model into the middle and lower atmosphere, J. Geophys. Res., 96, 1159-1172, 1991.

Heisenberg, W.: Zur statistichen Theorie der Turbulentz, Z. Phys., 124, 628-657, 1948.

Hines, C. O.: Internal atmospheric gravity waves at ionospheric heights, Can. J. Phys., 38, 1441-1481, 1960.

Hocking, W. K.: Observation and measurement of turbulence in the middle atmosphere with a VHF radar, J. Atmos. Terr. Phys., 48, 655-670, 1986.

Hocking, W. K.: The dynamical parameters of turbulence theory as they apply to middle atmosphere studies, Earth Planet Space, 51, 525-541, 1999.

Hodges Jr., R. R.: Generation of turbulence in the upper atmosphere by internal gravity waves, J. Geophys. Res., 72, 34553458, 1967.

Hodges Jr., R. R.: Eddy diffusion coefficients due to instabilities in internal gravity waves, J. Geophys. Res., 74, 4087-4090, 1969.

Justus, C. G.: Upper atmospheric mixing by gravity waves, Proceedings of the AIAA/AMS International Conference on the Environmental Impact of Aerospace Operations in the High Atmosphere, paper No. 73-495, Denver, CO, June 11-13, 1973.

Kelley, M. C., Kruschwitz, C. A., Gardner, C. S., Drummond, J. D., and Kane, T. J.: Mesospheric turbulence measurements from persistent Leonid meteor train observations, J. Geophys. Res., 108, 8454, doi:10.1029/2002JD002392, 2003.

Larsen, M. F.: Winds and shears in the mesosphere and lower thermosphere: Results from four decades of chemical release 
wind measurements, J. Geophys. Res., 107, SIA28.1-SIA28.14, doi:10.1029/2001JA000218, 2002.

Lindzen, R. S.: Thermally driven diurnal tide in the atmosphere, Q. J. Roy. Meteorol. Soc., 93, 18-42, 1967.

Lübken, F. J.: Seasonal variation of turbulent energy dissipation rates at high latitudes as determined by in situ measurements of neutral density fluctuations, J. Geophys. Res., 102, 1344113456, 1997.

Lübken, F. J., Hillert, W., Lehmacher, G., and von Zahn, U.: Experiments revealing small impact of turbulence on the energy budget of the mesosphere and lower thermosphere, J. Geophys. Res., 98, 20369-20384, 1993.

Medvedev, A. S. and Klaassen, G. P.: Vertical evolution of gravity wave spectra and the parameterization of associated wave drag, J. Geophys. Res., 100, 25841-25853, 1995.

Medvedev, A. S. and Klaassen, G. P.: Thermal effects of saturating gravity waves in the atmosphere, J. Geophys. Res., 108, 4040, doi:10.1029/2002JD002504, 2003.

Rees, D., Roper, R. G., Lloyd, K. H., and Low, C. H.: Determination of the structure of the atmosphere between 90 and $250 \mathrm{~km}$ by means of contaminant releases at Woomera, May 1968, Philos. T. Roy. Soc. London A, 271, 631-663, 1972.

Roble, R. G.: Energetics of the mesosphere and thermosphere, Geophys. Monogr., 87, 1-21, 1995.

Shimazaki, T.: Effective eddy diffusion coefficient and atmospheric composition in the lower thermosphere, J. Atmos. Terr. Phys., 33, 1383-1401, 1971.

Szewczyk, A., Strelnikov, B., Rapp, M., Strelnikova, I., Baumgarten, G., Kaifler, N., Dunker, T., and Hoppe, U.-P.: Simultaneous observations of a Mesospheric Inversion Layer and turbulence during the ECOMA-2010 rocket campaign, Ann. Geophys., 31, 775-785, doi:10.5194/angeo-31-775-2013, 2013.
Vlasov, M. N. and Kelley, M. C.: Estimates of eddy turbulence consistent with seasonal variations of atomic oxygen and its possible role in the seasonal cycle of mesopause temperature, Ann. Geophys., 28, 2103-2110, doi:10.5194/angeo-28-2103-2010, 2010.

Vlasov, M. N. and Kelley, M. C.: Eddy turbulence, the double mesopause, and the double layer of atomic oxygen, Ann. Geophys., 30, 251-258, doi:10.5194/angeo-30-251-2012, 2012.

Vlasov, M. N. and Korobeynikova, T. V.: Effect of the turbulence, infrared radiation and mass-averaged transport on the height distribution of temperature in the middle-latitude and high latitude thermosphere, Cosmic Res., 29, 469-474, 1991.

von Zahn, U., Lübken, F.-J., and Pfitz, C.: BUGATTI experiments: Mass spectrometric studies of lower thermosphere eddy mixing and turbulence, J. Geophys. Res., 95, 7443-7465, 1990.

Weinstock, J.: Nonlinear theory of acoustic-gravity waves, 1, Saturation and enhanced diffusion, J. Geophys. Res., 81, 633-652, 1976.

Weinstock, J.: Vertical turbulent diffusion in a stably stratified fluid, J. Atmos. Sci., 35, 1022-1027, 1978.

Weinstock, J.: Energy dissipation rates of turbulence in the stable free atmosphere, J. Atmos. Sci., 38, 880-883, 1981.

Weinstock, J.: Gravity wave saturation and eddy diffusion in the middle atmosphere, J. Atmos. Terr. Phys., 46, 1069-1082, 1984.

Yu, J. R. and She, C. Y.: Climatology of midlatitude mesopause region observed by a lidar at Fort Collins, Colorado $\left(40.6^{\circ} \mathrm{N}\right.$, $105^{\circ}$ W), J. Geophys. Res., 100, 7441-7452, 1995. 